\title{
국가기초구역기반 의사결정지원시스템 구축전략 \\ Strategic Plan for Building of Spatial Decision Support Service based on National Basic Districts
}

\author{
강 영 옥* \\ 강 애 띠 ** \\ Young Ok Kang \\ Ae Tti Kang
}

\begin{abstract}
요 약 공간적 의사결정지원시스템을 이용하여 합리적 의사결정을 성공적으로 이끌어내기 위해서는 오랜 기간 축적된 사람, 건물, 토지, 사업체 등에 대한 공간정보가 필요하다. 해외에서는 공간의 최소단위 를 정의하고 이 기반으로 정보를 구축하여 공간적 의사결정서비스에 활용하고 있다. 이러한 목적으로 우 리나라에서도 국가기초구역이 고시되었지만 국가기초구역의 활용도를 높이기 위해서는 속성정보의 구축 이 미비한 실정이다. 본 연구는 국가기초구역단위 공간데이터 축적을 통해 국가기초구역기반 의사결정지 원시스템을 구축하는 전략제안을 목표로 한다. 전략은 정보시스템 구성요소에 맞도록 제도부문, 데이터 및 시스템 부문, 서비스 부문으로 나누어 제시되었으며, 이를 통해 국가의 최소 공간단위로서 국가기초구 역의 역할이 더욱 견고해질 것으로 기대한다.
\end{abstract}

키워드 : 국가기초구역, 의사결정지원시스템, 공간적 의사결정지원시스템

\begin{abstract}
The accumulation of spatial information - people, building, land and businesses- is required to make a rational decision successfully by utilizing the Spatial Decision Support Systems (SDSS). Many foreign countries have utilized the SDSS by defining the minimum unit of spatial area and implementing the spatial database based on it. Even though in Korea, National Basic Districts were created for the purpose of utilizing these districts as a basic area, they have not been utilized as a basic area of managing the spatial database due to the lack of attributes data. Therefore, the goal of this research is to suggest the strategies of building Spatial Decision Support Systems through the accumulation of the spatial data based on National Basic Districts. We propose the strategies in three different fields - legal system, database and application, services. We expect that the role of National Basic Districts will be strengthened by executing our strategies as a minimum spatial unit of Korea.
\end{abstract}

Keywords : National Basic Districts, Decision Support System, Spatial Decision Support System

\section{1. 서 론}

국가기초구역은 기존의 행정읍면동 보다 규모가 작으면서 동질적이고 경계변화가 작은 국가의 최소 공간단위를 목표로 설정되었다[6]. 국가기초구역은 도로, 하천, 철도 등 뚜렷한 지형지물을 이용한 경 계 설정, 생활권 중심 경계설정을 원칙으로 하여 전 국의 지역을 구분하였으며, 2012년 12월 23일자로
전국에 총34,019개가 고시되었다. 2013년 말까지 국 가기초구역 단위로 각종구역 고시가 이루어지고, 2014년부터 본격적으로 국민들의 생활에 활용될 예 정이다.

국가기초구역의 핵심가치는 각종구역 설정의 최 소단위로서 뿐 아니라 다양하게 산출되는 국가기초 구역기반 공간데이터를 이용하여 공공 및 민간의 다양한 공간의사결정지원이 이루어질 수 있도록 하

\footnotetext{
+ This research was supported by a grant from a Strategic Research Project(Strategic Plan for Improvement of Data Model of National Basic Districts and Building of Decision Support System based on National Basic Districts) funded by the Korea Information Research and Development Institute(KLID).

Young Ok Kang, Professor, Dept. of Social Studies(Geography), Ewha Womans University, ykang@ewha.ac.kr (Corresponding Author)

*Ae Tti Kang, Ph.d. student, Dept. of Social Studies(Geography), Ewha Womans University, aetti@naver.com
} 
는 것이다.

본 연구의 목적은 국가기초구역 기반의 공간적 의사결정지원을 가능케하는 서비스 제공 전략 수립 에 있다. 이를 위해 첫째, 의사결정지원시스템 구축 의 구성요건과 유용성을 확인하고, 국가적 차원에서 진행되는 공간의사결정지원시스템 구축 현황 및 활 용의 문제점을 분석하고자 한다. 둘째, 국가기초구 역사업의 추진현황과 관리시스템 구축현황을 분석 하며, 이와 함께 국가기초구역과 연계 가능한 국가 적 차원에서 추진되고 있는 중요사업들을 분석한다. 이를 바탕으로 셋째, 국가기초구역 기반의 공간의사 결정지원 서비스 제공을 위한 제반전략을 제시하고 자 한다.

\section{2. 이론적 배경}

\section{1 의사결정지원시스템의 개념 및 활용}

의사결정지원시스템(Decision Support System)은 경영분야에서 의사결정을 지원하기 위한 개념에서 시작되었다. 경영학에서의 의사결정지원시스템은 경 영활동에 있어 의사결정자의 비구조적, 반구조적 문 제를 해결하기 위해 정보시스템의 데이터와 의사결 정모델 그리고 인터페이스를 통해 의사결정능력을 지원해주는 정보시스템이라고 정의하고 있다. 의사 결정지원시스템은 자료, 모델, 지식베이스로 구성된 시스템이 사용자 인터페이스를 통해 의사결정자와 상호작용하는 시스템이라고 볼 수 있다 [3, 26, 30].

의사결정지원시스템의 데이터베이스와 지식베이 스는 회사업무 진행과정에서 축적될 수 있는 것이

Table 1. Analysis Types of a Decision Support System [26]

\begin{tabular}{|c|l|}
\hline $\begin{array}{c}\text { Types } \\
\text { Analysis } \\
\text { model }\end{array}$ & $\begin{array}{l}\text { Model to observe the changes in } \\
\text { the values of other variables by } \\
\text { varying the variable }\end{array}$ \\
\hline $\begin{array}{c}\text { Sensitivity } \\
\text { Analysis model }\end{array}$ & $\begin{array}{l}\text { Model to observe the changes of } \\
\text { the other variables as a result of } \\
\text { the changes of one value }\end{array}$ \\
\hline $\begin{array}{c}\text { Target Pursuit } \\
\text { Model }\end{array}$ & $\begin{array}{l}\text { Model to find the valiables that } \\
\text { can be achieved the target }\end{array}$ \\
\hline $\begin{array}{c}\text { Optimization } \\
\text { Model }\end{array}$ & $\begin{array}{l}\text { Analytical model to obtain the } \\
\text { optimal value of one or more } \\
\text { target variables when Specific } \\
\text { constraints are given }\end{array}$ \\
\hline
\end{tabular}

지만 모델베이스는 회사업무의 전문가가 업무를 분 석하여 설계해야 한다. 일반적으로 의사결정지원시 스템의 모델은 가정 분석 모형, 민감도 분석 모형, 목적추구 분석 모형, 최적화 분석 모형의 4 가지로 유형화할 수 있다. Table 1은 의사결정지원시스템 의 모델을 유형별로 설명한 것이다 [26].

이혜영과 박석순 [14]은 “오염총량관리를 위한 의 사결정지원시스템 적용”에서 경안천의 수리 데이터 베이스를 활용하고 유역모델과 하천모델을 적용한 의사결정지원시스템인 WARMF를 활용하여 목표 수질을 높이기 위한 수문학적 요구치를 산정하였다. 박기용, 안성식, 이훈영 [28]은 “의사결정지원시스템 을 이용한 외식산업 편익세분화에 관한 연구”에서 외식기업의 비용편익을 데이터베이스화하고 업무모 델을 통계적으로 구축하여 외식기업의 성공적인 마 케팅전략에 필요한 기초연구를 수행한 바 있다.

최근에는 웹기반 의사결정지원시스템과 빅데이터 를 활용한 의사결정지원시스템이 구축되는 추세이 다. 이훈영, 임민형, 양주환 [4]은 “웹기반 고객효용 측정시스템에 관한 연구”에서 인터넷 등 정보기술 을 이용하여 고객과 커뮤니케이션함으로써 그 효용 을 측정하여 이를 마케팅 의사결정에 활용할 수 있 는 의사결정지원시스템을 설계하였다. 미국의 경우 국립보건원에서 유전자 데이터공유를 통해 질병치 료체계를 마련하고 있으며, 미국 퇴역군인의 전자의 료기록 분석을 통해 맞춤형 의료서비스를 지원하고 있다. 한국의 경우 한국석유공사에서 빅데이터를 이 용하여 유가사재기 등을 예측하고 공급량을 결정하 는 등의 의사결정지원시스템을 활용하고 있다 [24]. 그러나 의사결정지원시스템을 성공적으로 활용하 기 위해서는 모델과 관련된 데이터베이스의 구축이 필수적이다. 이혜영과 박석순 [14]은 오염총량관리 를 위해 환경부에서 구축한 오염원데이터베이스를 활용하였고, 박기용, 안성식, 이훈영 [28]은 패밀리 레스토랑을 이용한 경험이 있는 남·녀 대학생 및 대학원생, 일반인과 패밀리레스토랑에 대한 기본적 이해를 가진 사람들에 대한 설문 조사 결과 데이터 베이스를 구축하여 외식사업의 편익분석에 활용하 였다. 이훈영, 임민형, 양주환 [13]은 인터넷을 통한 설문조사방법으로 고객에 대한 정보를 데이터베이 스화하여 고객효용을 시률레이션 하였다. 또한 미국 의 질병치료체계 수립에 사용된 유전자데이터베이 스나 한국석유공사의 석유공급·수요데이터베이스 또 
한 모델에 맞게 수집된 데이터베이스가 존재하기 때문에 가능했다.

\section{2 공간적 의사결정지원시스템의 활용}

'21세기 고도화사회에 대비하여 국가차원의 GIS 기반 조성'과'국가공간정보기반 확충’을 계획기조로 삼았던 제 1 차 국가GIS사업과 제 2 차 국가GIS사업이 완료되면서 기본지리정보가 구축되었고, GIS활용체 계구축의 기반이 마련되었다. 이어 2005년부터 2009 년까지 진행된 제 3 차 국가GIS사업을 통해 국가통계 지식체계, 공간영상정보구축, 국가기본도 수정갱신 및 제작, 해양기본도 제작, 전자해도 제작, 해안선조 사 등 기본지리정보가 구축되었고 이를 위한 표준 화조사와 기술개발, 활용 및 유통부문사업이 진행되 면서 국가GIS사업은 점차 공간데이터 구축에서 공 간데이터 활용의 패러다임으로 넘어가고 있다 [19].

이렇게 공간데이터가 풍부해지면서 이를 활용하 는 지식베이스와 업무모델이 존재하는 분야에서 공 간적 의사결정지원시스템에 대한 연구가 활발히 진 행되고 있다. 임은선 [4]은 “생활폐기물관리를 위한 공간적 의사결정지원시스템 구축에 관한 연구”에서 기본지리정보인 건물에 폐기물 배출량을 입력하였 고, 도로망도와 건물도를 결합하여 폐기물 수집구역 을 구축하였다. 이건학, 신정엽, 신성희[12]는 인천 시를 사례로 한“GIS 기반의 다기준 의사결정분석 기법을 이용한 시립 미술관 입지 적합성 분석 연 구”에서 교통접근성을 분석하기 위해 도로망도를 이용했고, 인구잠재성을 분석하기 위해 동별 인구수 를 이용하였으며, 환경적 형평성을 분석하기 위해 토지피복분류도와 측정위치별 대기오염데이터를, 경제적 형평성을 분석하기 위해 구별 경제규모와 고용정도 등 다양한 공간데이터와 속성정보를 결 합하였다.

공간데이터와 모델을 이용한 공간적 의사결정지 원시스템에 대한 연구는 학술적 측면에서 뿐만 아 니라 실제 시스템이 구축되고 이를 활용하는 국내 외 사례가 다수 존재한다. 대표적인 외국의 공간적 의사결정지원시스템인 미국의 UrbanSim은 토지이 용, 교통 및 환경계획(대기의 질)수립 지원을 위해 1996년부터 개발되었으며 1998년부터 서울, 암스테 르담, 브뤼셀, 디트로이트, 호놀루루, 휴스턴, 파리, 로마, 솔트레이크시티, 샌프란시스코, 시애틀, 타이 베이, 텔아비브, 취리히 등에서 이를 도입하기 시작
하였다. UrbanSim 모형은 크게 가구와 정보, 부동 산개발자와 경제활동으로 구성되어 있으며 도시계 획과 관련된 각 주체들의 활동을 모형화하여 특정 시나리오하에 어떻게 상호작용하는지 시뮬레이션해 주는 도구라고 볼 수 있다 [7].

국토교통부는 2006년부터 2009년까지 4개년간 국 토계획수립을 위한 의사결정지원시스템인 국토공간 계획지원체계(KOrea Planning Support Systems, 이하 KOPSS)를 구축하였다 [18]. KOPSS는 국가 공간정보통합체계에서 관리하는 기본공간정보 및 용도지역지구, 기타지형지물과 주제도 등을 활용하 여 국토계획의 모델별로 데이터마트를 구성한 후 공간적 의사결정에 활용하는 시스템이다. Figure 1 은 KOPSS에서 지자체 정보시스템의 자료는 온라 인으로 수집하고, 타 기관 $\mathrm{DB}$ 는 오프라인으로 수집 하여 데이터마트를 구축하는 과정을 나타낸 것이다. KOPSS 외에도 서울시 SDW(Spatial Data Warehouse), 통계청의 통계네비게이터, 소상공인진흥원의 상권 정보시스템, 지오비전의 의사결정지원 시스템 등이 관련분야 의사결정지원을 지향하며 시스템이 구축 되어 있다.

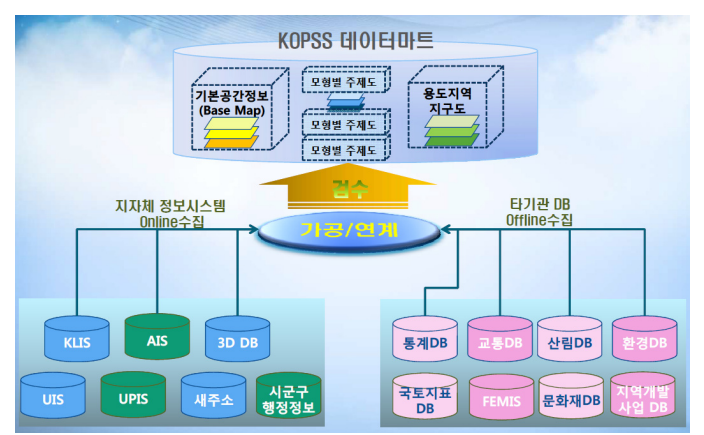

Figure 1. Datamarts Construction Process of KOPSS [18]

이렇듯 공간적 의사결정지원 연구 및 시스템 구 축에 있어 기본적인 공간데이터의 구축이 필수적이 며 이를 위해 국가주도의 공간데이터 구축이 이루 어져 왔지만 보다 상세한 수준에서의 의사결정지원 을 하기에는 문제가 있었다. 상기한 임은선[4]은 논 문에서 폐기물 수집구역을 구축하는데 있어 폐기물 에 대한 정보가 행정동 단위를 최소 단위구역으로 정의하고 있기 때문에 자세한 수준에서 의사결정지 원이 어려웠고 그래서 자체적으로 폐기물 수집구역 
을 정의하여 구축하였다. 이건학, 신정엽, 신성희 [12]는 논문에서 인구잠재성을 측정하는데 있어 행 정동 단위 정보밖에 입수할 수 없어 동 단위 인구 분포 대신 지적 데이터를 이용하여 매우 상세한 스 케일에서 인구분포를 추정했다고 하였다. 인구정보 를 대체지수로 대체한 것보다 더 문제는 경제적 형 평성을 구하는 것이었는데 경제적 형평성을 구성하 는 고용, 사업체수 등의 정보가 구별로 집계되기 때 문에 결국 미술관의 입지적합성이 구별 스케일에서 결론이 나는 결과가 도출되었다.

공간적 의사결정지원시스템인 $\mathrm{KOPSS}$ 의 경우도 지역계획지원모형인 “지역이”의 경우 지역균형개발 등에서 시계열 매핑을 할 때 시계열 데이터가 제공 되는 단위가 시군구밖에 존재하지 않아, 지역균형개 발의 공간분석이 시군구스케일에서 분석되는 제한 점을 갖고 있다. 또한 Figure 2와 같이 토지계획지 원모형인 “터잡이”는 기본적으로 중첩분석의 원리 를 사용하고 있는데, 중첩분석은 입력되는 데이터의 축적이 다르면 소축적에서 입지가 선정되는 문제점 이 있다. 또한 민간에서는 국가에서 제공되는 데이 터의 최소공간단위로서는 분석의 의미가 희석되기 때문에 나름대로의 기준으로 별도로 구역을 설정하 여 활용하는 현상을 낳고 있다.

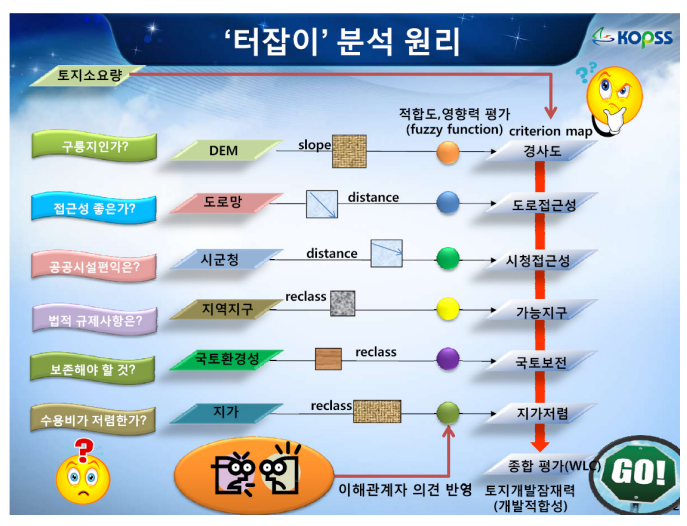

Figure 2. Spatial Analysis Module of KOPSS called Terjabi [17]

\section{3. 공간정보관리 트랜드와 국가기초구역}

\section{1 공간정보관리의 트랜드}

최근 공공정보의 공개 및 공유가 국가서비스의 중요한 화두가 되면서 국가가 보유하고 있는 정보
의 공개 및 공유사례가 많아지고 있는데 국외의 경 우 원(raw)데이터를 공개하여 활용범위를 넓이고 있는 것이 특징이다. 미국은 Data.gov(2009.5) 사이 트[34]를 개설하여 공공정보를 제공하고 있으며, 2012년 12월 현재 378,529개의 원(raw)데이터와 공 간 데이터셋이 제공되고 있다. 정보공개기관은 지속 적으로 확대되어 180 개 주무부처와 산하기관의 정 보가 공개되고 있고, 데이터의 활용, 접근성에 기초 해 분류된 애플리케이션은 정부 1,264 개, 시민개발 236 개, 이동통신용 103 개가 개발 운영되고 있다.

영국은 2010년 Data.gov.uk[32]을 개발해 스마트 정부(smart government)가 되기 위한 전략으로 부 처 및 기관이 생성하고 보유하고 있던 도로, 교통, 항만, 범죄, 재난재해, 주택, 환경, 의료 등에 대한 데 이터베이스를 구축하고 공개하였으며, 현재 6,000 개 의 정부 공공기관의 데이터 세트에 접근할 수 있는 단일창구로 기능하고 있다. OGL(Open Government Licence)을 도입해 다수의 정부 데이터를 재사용할 수 있도록 포괄적인 허가 절차를 마련했으며, $\mathrm{PDP}($ Public Data Principles)를 수립, 어떤 정부 데 이터를 어떻게 공개할 것인지에 대한 원칙을 수립 하였다. 또한, 얼마나 데이터가 재사용 가능하며, 어 느 정도로 공개되었는지를 심사해 평가하는 평가체 계도 구축하고 자료생성 기관별, 각 연방 구성 국가 별, 표제어별로 정보를 제공하고 있다.

국내의 경우 2008년 11월 수립된 '국가정보화 기 본계획'과 2010년에 수립한 '공공정보 민간 활용촉 진 종합계획'을 토대로 공공정보 개방 및 활용정책 의 제도적 기반을 마련하고 있지만 국외와 같이 원 (raw)데이터보다는 오픈API를 이용한 공개가 주를 이루고 있다. '공유자원포털'[13]은 국가가 보유하고 있는 공공정보를 활용하여 다양한 서비스를 제공하 고 있다. 서비스(Open API), 소프트웨어, 컴포넌트 형태의 국가공유자원에 대한 검색/활용을 지원하는 프로그램 제공 서비스와 데이터 형태의 국가공유자 원에 대한 검색/활용을 지원하는 데이터 제공 서비 스로 구성되어 있으며 현재 18 개 기관 7,752 종의 데 이터를 보유하고 있다.

서울시가 운영하는 '열린 데이터 광장'[29]은 서울 시가 가진 공공데이터를 공개하기 위해 2012년 5월 에 시작한 웹사이트로 원천데이터의 다운로드와 Open API를 통한 서비스를 제공하고 있다.

공간데이터 공개현황을 알아보기 위해 통계 및 
공간정보를 제공하는 기관별 최소공간단위를 조사 한 결과는 Table 2 에 정리되어 있다. 대부분의 기관 에서 변동가능성이 많은 행정동을 사용하거나 공간 단위가 커서 대표치로서 의미가 희석되는 시도 및 시군구를 사용하고 있었고, 통계청만이 집계구단위 로 자료를 제공하고 있었다. 그러나 집계구의 경우 일부 경계가 매년 변경되며 통계청 내에서만 사용 되고, 타 기관에서 집계구 단위로 정보를 제공하지 않아 최소단위지역으로서의 의미가 부족하다[3].

외국의 경우 국가적 차원에서 최소 공간단위를 설정하고, 그 단위로 관련 통계자료를 제공하는 경 우가 많다. 미국의 경우 미국 통계청(United States Census Bureau)에서 10년마다 발표되는 센서스조 사 결과는 국토구역의 최소구획단위인 블록이나 센 서스트랙단위로 제공되므로 연구자들은 블록이나 블 록그룹, 센서스 트랙단위 속성정보를 구하여 원하는 스케일의 구역단위로 재생산이 가능하다(Figure 3).

또한 주정부와 산하의 교육, 선거, 도시 관리구역 등의 정보를 Blocks을 통합한 단위로 공표하여 다양한 위계의 자료 간 연계활용이 용이하게 하고 있다[3].

Table 2. Minimum Area Units of Spatial information offered by Public Institution

\begin{tabular}{|c|c|}
\hline Service and Statistics & $\begin{array}{l}\text { Minimum Area } \\
\text { Units }\end{array}$ \\
\hline $\begin{array}{c}\text { Statistics Nagivator(National } \\
\text { Statistical Office) }\end{array}$ & $\begin{array}{c}\text { Administrative } \\
\text { Dong and Census } \\
\text { Output Area }\end{array}$ \\
\hline $\mathrm{e}^{-}$National Indicators & $\begin{array}{l}\text { Administrative } \\
\text { Si and Do }\end{array}$ \\
\hline $\begin{array}{c}\text { Local Administration } \\
\text { Integrated Information } \\
\text { System }\end{array}$ & $\begin{array}{l}\text { Administrative } \\
\text { Si, Gun and Gu }\end{array}$ \\
\hline $\begin{array}{c}\text { Statistical Information on } \\
\text { Land and Transport }\end{array}$ & $\begin{array}{l}\text { Administrative } \\
\text { Si and Do }\end{array}$ \\
\hline National Atlas & $\begin{array}{l}\text { Administrative } \\
\text { Si, Gun and Gu }\end{array}$ \\
\hline National Fire Data System & $\begin{array}{l}\text { Administrative } \\
\text { Dong }\end{array}$ \\
\hline $\begin{array}{l}\text { Statistics of the Ministry of } \\
\text { Health and Welfare }\end{array}$ & $\begin{array}{l}\text { Administrative } \\
\text { Si, Gun and } \mathrm{Gu}\end{array}$ \\
\hline $\begin{array}{c}\text { Statistics of the Ministry of } \\
\text { Culture and Tourism }\end{array}$ & $\begin{array}{l}\text { Administrative } \\
\text { Si, Gun and Gu }\end{array}$ \\
\hline $\begin{array}{c}\text { Statistics of the National } \\
\text { Police Agency }\end{array}$ & $\begin{array}{l}\text { Administrative } \\
\text { Dong }\end{array}$ \\
\hline $\begin{array}{l}\text { Statistics of Ministry of } \\
\text { Education and Science }\end{array}$ & School \\
\hline $\begin{array}{l}\text { Statistics of the National } \\
\text { Election Commission. }\end{array}$ & $\begin{array}{l}\text { Administrative } \\
\text { Dong }\end{array}$ \\
\hline
\end{tabular}

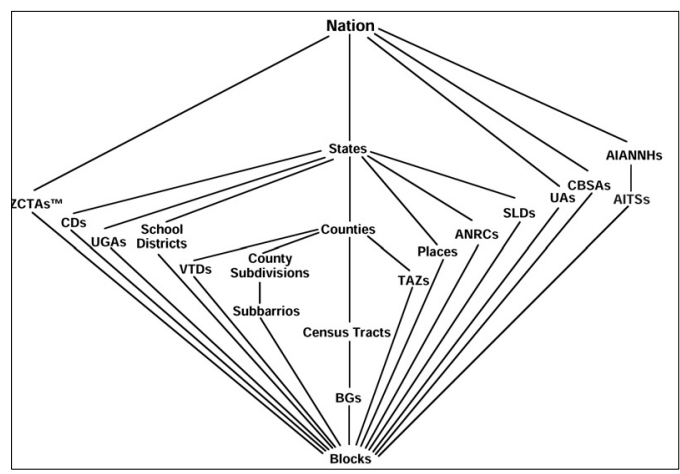

Figure 3. Topological Relationships of America's Statistical Area

\section{2 국가기초구역의 구획과 관리시스템 구축}

우리나라도 국가 및 지방자치단체 등에서 수많은 전산화된 정보들을 공개하고 있지만 Table 3 의 조 사결과와 같이 제공단위가 통일되지 않고, 제공하는 공간단위가 커서 자료들을 공간적 의사결정지원에 활용하기에는 큰 어려움이 있다. 따라서 작은 단위 의 공간적 기본단위를 정하고 이를 공간정보 제공 의 통일된 단위로 정할 필요가 있다. 이를 위해 국 가에서는 국가기초구역을 구획하고 이를 관리하는 제도를 도입하였다.

국가기초구역은 철도, 하천, 도로 등 지형지물과 행정경계를 고려하여 생활권 중심으로 설정한다는 목표 하에 경계가 설정되었으며 구역 하나하나에 5 자리 숫자의 번호가 부여되어 있으며, 하나의 행정 동이 평균 10 여개 정도로 분할되어 있는 규모이다 [Figure 4]. 2013년 학교, 우편, 소방, 경찰구역 등이 국가기초구역 단위로 구역을 고시하도록 「도로명 주소법」제 8 조의 3 에 규정되어 국가기초구역의 사용 에 대한 제도가 만들어진 상태이다.

국가기초구역의 관리를 위해 국가기초구역관리시 스템이 구축되었으며 국가기초구역의 관리, 대민서 비스 등을 수행하고 있다. 국가기초구역관리시스템 은 국가기초구역의 고시 등 실제적인 기초구역업무 지원을 위한 기초구역관리프로그램, 기초구역을 활 용하는 기관들의 각종구역설정을 돕기 위한 기초구 역 웹서비스, 국민들이 기초구역을 조회하고 활용할 수 있도록 각종 정보를 공개하는 기초구역 대민서 비스로 구성되어 있다. 국가기초구역관리시스템은 국가 주소정보시스템 내에서 운영되며 안전행정부 의 주민등록전산과 국토해양부의 KLIS와 연계되어 
주민정보와 연속지적을 활용할 수 있도록 되어 있 다 $[10,11]$.

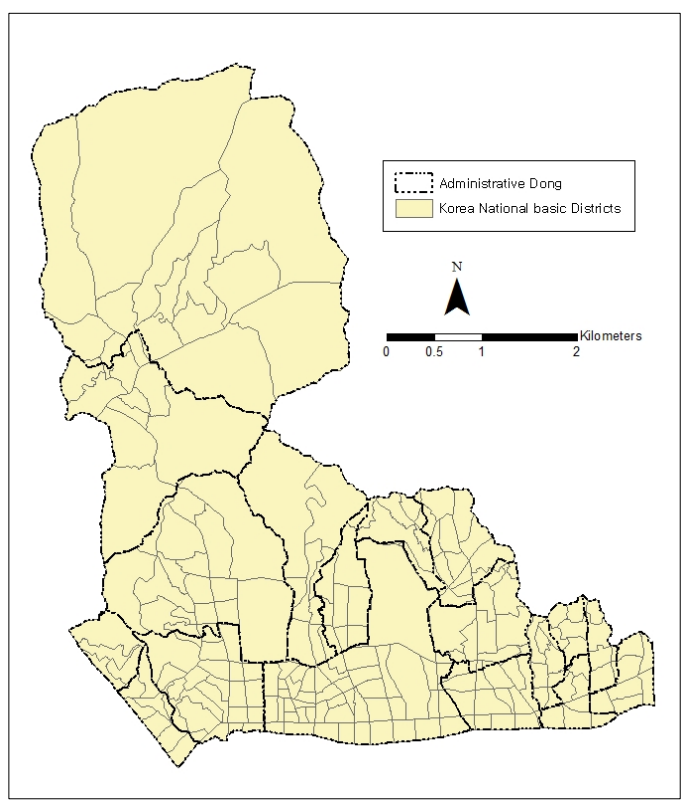

Figure 4. Comparison of National Basic

Districts and Administrative

Dong (Jongrogu, Seoul)

현재 국가기초구역은 속성정보로 면적, 고시일자, 기초구역번호, 행정읍면동코드 등을 포함하고 있으 며 국가기초구역 구획에 참조자료로 사용된 인구수 만을 관리하고 있다. 따라서 국가기초구역의 구획취 지에 맞는 활용을 위해서는 현재 관리하고 있는 인 구 수 뿐만 아니라 국가기초구역기반의 속성정보를 확대 구축하여 명실상부 국가의 최소공간단위로 자 리매김하고 다양한 공간적 의사결정의 기초가 되도 록 할 필요가 있다.

\section{3 국가기초구역과 연계가능한 시스템 현황}

현재 국가기초구역관리시스템은 안전행정부가 관 리하는 주민전산시스템과 연계되어 주기적으로 기 초구역별 주민인구수를 갱신하는 체계를 가지고 있 다. 국가차원의 공간정보관리시스템들이 공간정보 의 구축단계를 넘어서 공간정보의 통합, 활용의 단 계로 접어들고 있는 만큼 연계를 통해 국가기초구 역단위 공간정보구축이 가능한 시스템들이 다수 존 재한다.
국토교통부의 국가공간정보통합체계는 각 기관에 서 관리하고 있는 공간정보를 범국가적인 차원에서 연계·통합하고 이를 행정업무와 대민서비스에 공동 으로 활용하기 위한 정보시스템이다[16]. 각 기관에 서 구축한 기본공간정보, 기타지형지물, 행정주제정 보, 개발 및 보전 관련 주제를 보유하고 있다. 각 기관에서 보유한 데이터를 국가 공간정보 통합체계 $\mathrm{DB}$ 서버에서 저장하는 방식으로 공간데이터를 관리 하는데 $\mathrm{DB}$ 연동, 파일연동, 웹 서비스 등의 방식으로 연계한다. 2011년 말 현재 13 개 기관의 44개 시스템 과 연계되어 있는데 중앙부처 및 공공기관 20개 시 스템의 공간정보를 한 곳에서 볼 수 있도록 국가공 간정보통합체계에 연계·공유하고 국가공간정보통합 체계에 구축한 기본공간정보와 변동자료 등을 해당 부처 시스템에 실시간 제공하고 있다 (Figure 5).

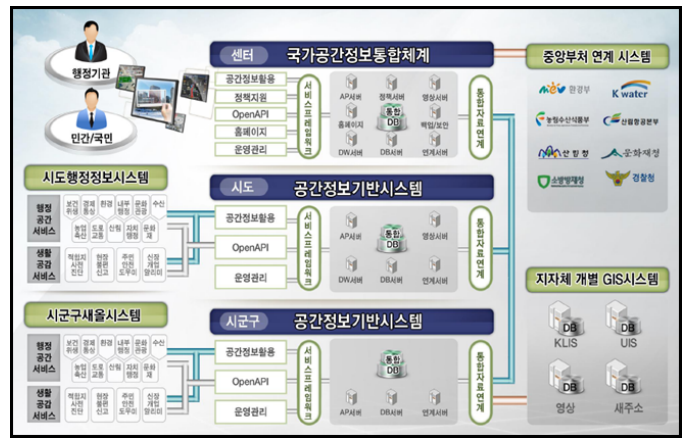

Figure 5. Concept of National Spatial Data Infrastructure Information System

행정공간정보체계[23]는 시도·시군구의 행정정보 시스템에 공간정보를 접목시켜 자치단체 업무 담당 자는 효율적 행정업무 지원을, 지역주민은 생활에 밀착된 대민 서비스를 제공받을 수 있도록 도와주 는 시스템이다. 행정공간정보체계는 시군구행정정 보시스템(새올)에 대국민지도서비스(생활공감 지도 서비스)가 결합된 형태를 취하고 있다(Figure 6). 대국민지도서비스의 경우 안전행정부 대표 사이트 를 구축하여 인허가자가진단, 생활불편신고, 공공서 비스안내지도, 스마트안전귀가 등의 서비스를 제공 하고 있다.

국가공간정보체계와 행정공간정보체계는 공간정 보와 행정에서 발생되는 다양한 데이터의 통합체계 로서 중요성을 지니지만 아쉽게도 국가공간정보체 


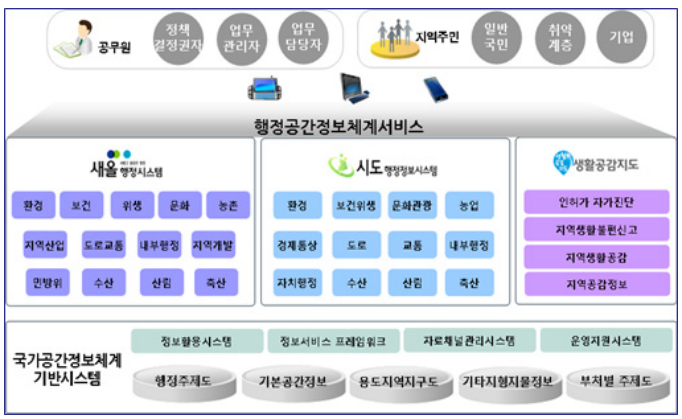

Figure 6. Concept of Administrative-Spatial information System

계 내에는 건축물에 대한 상세정보와 토지에 대한 상세정보는 연계되어 있지 않다. 이를 위해서는 별 도의 시스템 연계에 대한 고려가 필요하다. 즉 건축 물대장은 건축물에 대한 다양한 정보를 담고 있는 데 건축물대장은 국토교통부의 건축행정시스템(세 움터)에서 관리하고 있다. 건축행정시스템은 건축허 가부터 착공, 사용승인에 이르는 건축행정업무와 주 택조합설립부터 사업승인, 사용검사에 이르는 주택 행정업무의 전 과정을 전자적으로 처리하고, 사용승 인과 동시에 건축물대장을 비롯한 각종 문서를 자 동으로 생성하는 시스템이다. 건축행정시스템은 총
괄표제부, 대장동별개요, 대장층별개요, 부속지번현 황 등에 대한 정보를 관리하는데 이중 건축물대장 총괄표제부에는 위반건축물구분, 대지면적, 건축면 적, 건폐율, 연면적, 용적률, 주건축물수, 부속건출물 수, 주용도코드, 세대수, 가구수, 총주차대수, 사용승 인일자 등 국가기초구역단위로 정보제공이 가능한 건물에 대한 정보를 보유하고 있다 [1].

또한 토지에 대한 정보는 한국토지정보시스템 (KLIS)에서 관리하고 있다. 한국토지정보관리시스 템에서 관리하는 연속지적과 연계된 토지거래, 개별 토지특성, 지가자료, 지적측량자료 등은 국가기초구 역단위로 정보제공이 가능하다.

\section{4. 국가기초구역기반 의사결정지원시스템 구축전략}

국가기초구역기반 의사결정지원시스템의 목표는 국가기초구역을 기반으로 다양한 통계자료, 행정자 료를 서비스하여 국토계획, 지역계획, 그리고 공간 단위로 이루어지는 여러 업무를 과학적이고 효율적 으로 지원하는 것에 목표를 두고 있다. 시스템 구축 전략은 내외부 현황 및 환경분석을 토대로 시사점

\section{Strategies}

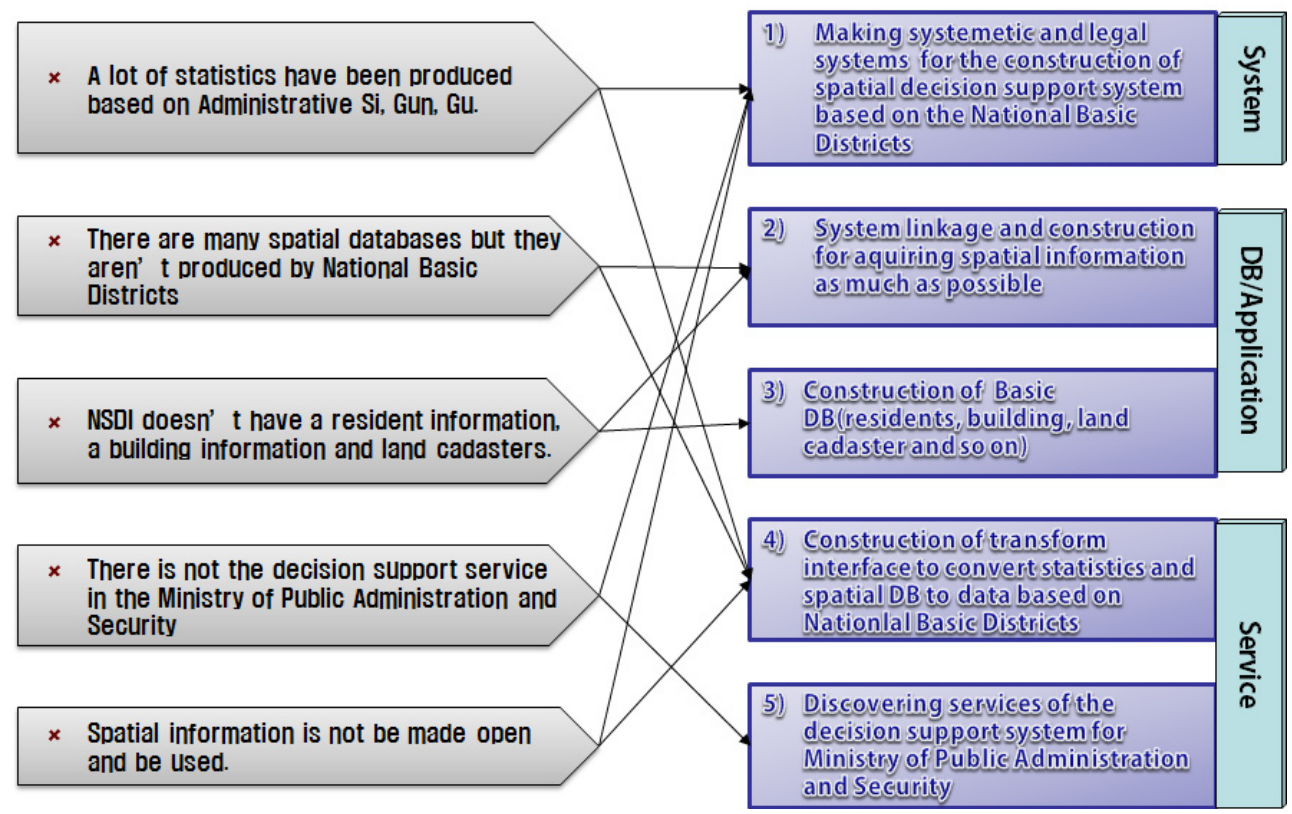

Figure 7. Tasks for the Decision Support Service based on National Basic Districts 
및 문제점을 분석하고, 이를 토대로 세부전략목표를 수립하였으며, 세부전략별 추진계획을 수립하였다 (Figure 7).

현황분석과 환경 분석을 통한 시사점 및 문제점 을 살펴보면 첫째, 많은 통계들이 시군구의 공간단 위로 산출되고 있으며, 둘째, 국가기초구역이 법적 으로 제도화되어 있으나 공간 $\mathrm{DB}$ 가 국가기초구역 으로 산출되고 있지는 않았다. 셋째, 국가의 거의 모든 공간정보 관련 시스템을 연계한 국가공간정보 통합체계에 공간의사결정의 기본이라 할 수 있는 주민정보, 건축물정보, 토지대장 등의 정보는 포함 되어 있지 않았다. 넷째, 안전행정부 업무에 대한 의사결정지원서비스가 구축되어 있지 않은 상태이 며 다섯째, 공간정보의 공개가 잘 이루어지지 않거 나 공개된 자료의 활용도가 떨어졌다.

이러한 문제점을 해결하기 위해 의사결정지원 시 스템 구성에 필요한 세가지 기본요소인 제도, 데이 터베이스 및 시스템아키텍쳐, 서비스 측면에서 전략 을 수립하였다. 제도측면에서는 기초구역단위 의사 결정지원체계 구축을 위한 제도적·법적기반 마련을, 데이터베이스 및 시스템 아키텍쳐 부문에서는 가능 한 많은 공간정보를 취득하기 위한 시스템 연계 및 기초구역기반의 기본 $\mathrm{DB}$ 구축이 필요할 것으로 판 단되었다. 서비스 측면에서는 기존 통계 및 공간DB 의 기초구역 단위 변환 인터페이스 구축과 안전행 정부 의사결정지원을 위한 서비스 발굴이 필요할 것으로 분석되었다.

현황분석을 통해 도출된 문제점들은 여러 측면의 전략으로 해결 가능한 것으로 보았다. 예를 들어 많 은 통계들이 시군구 등의 공간단위로 산출되어 지 역적 현황파악에 도움이 되지 못하고 있다는 문제 점은 제도적 측면에서 기초구역단위 의사결정지원 체계 구축을 위한 제도적·법적 기반을 마련해야 하 고, 서비스 측면에서는 기존 통계 및 공간DB의 기 초구역단위 변환 인터페이스를 구축해야 한다는 전 략을 통해 해결될 수 있다.

\section{1 제도마련}

국가기초구역 단위로 속성정보를 산출하기 위해 서는 국가기초구역을 이용하는 각종구역 관리기관 및 통계를 산출하는 국가기관들이 국가기초구역단 위로 속성정보를 산출할 수 있는 법적규정이 필요 하다. 국가기초구역과 관련된 현행법인 r도로명주
Table 3. Amendments of the Law to Build Spatial Decision Support Service based on National Basic Districts

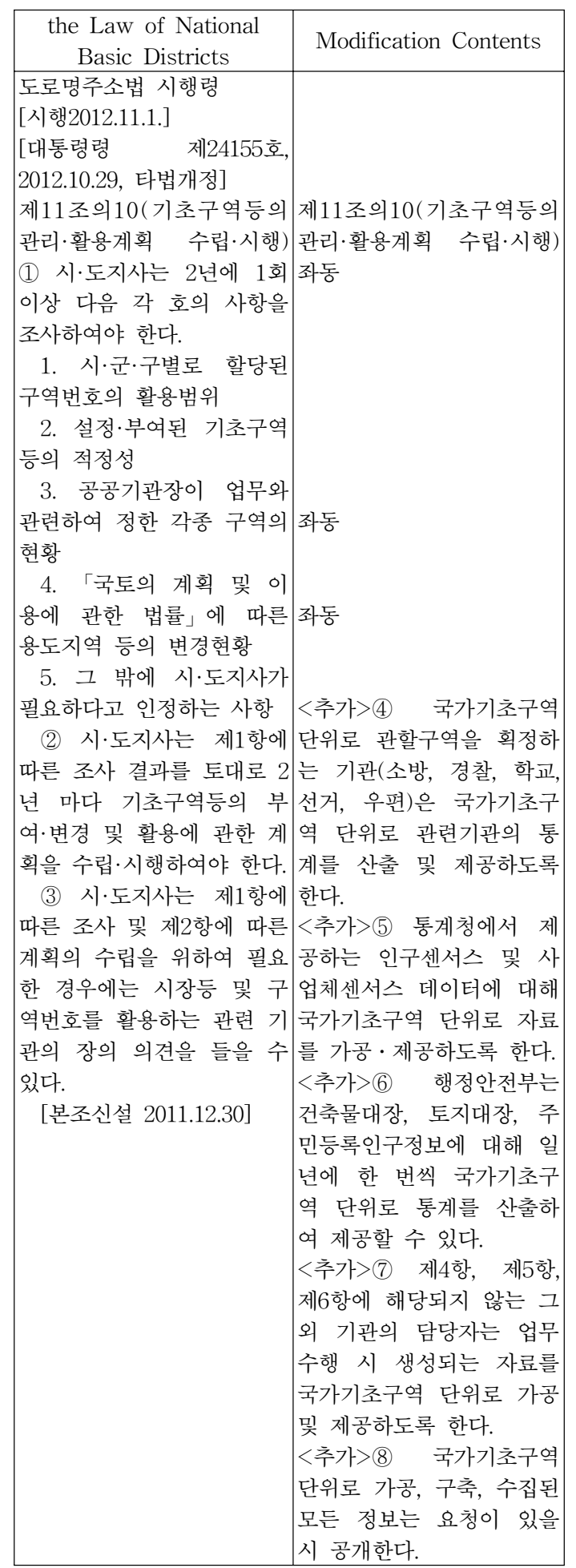


소법」과 r공공기관의정보공개에관한법률」을 고려하 여 도로명주소법에 기초구역 등의 관리·활용계획수 립·시행조항을 추가함으로써 각종구역관리기관, 통 계청, 행정안전부 등의 기관이 국가기초구역단위로 정보를 공개하도록 규정하는 것이 필요하다. 이와 관련하여 도로명주소법 시행령의 제 11 조 10 항에 관 련사항을 추가하는 것이 바람직할 것으로 판단되었 다(Table 3).

\section{2 국가기초구역기반 의사결정지원시스템에 활용 될 속성자료 가공}

국가기초구역기반 의사결정지원시스템에 활용될 속성자료는 기본 $\mathrm{DB}$, 기존 공간DB, 산출 $\mathrm{DB}$ 로 구분 하여 가공·관리하도록 할 필요가 있다 [Figure 8]. 기본 $\mathrm{DB}$ 란 국민들이 관심을 가지는 공간정보의 기 본이 되는 건축물정보, 토지정보, 사업체정보, 주민 정보를 기초구역단위로 구축한 $\mathrm{DB}$ 이며 주변 환경 을 이해하고 공간적 의사결정을 수립하는데 있어 기본이 된다. 따라서 국가기초구역의 속성정보 확장 의 차원에서 기본 $\mathrm{DB}$ 의 구축은 필수적이다. 기존 공 간DB는 기존 $\mathrm{GIS}$ 에서 관리하고 있는 공간DB중 기 초구역단위 의사결정지원서비스에 필요하여 기초구 역단위로 변환한 $\mathrm{DB}$ 를 의미한다. 기존 공간DB가 풍부해질수록 보다 상세한 차원에서 지역에 대한 탐색이 가능해진다. 산출 $\mathrm{DB}$ 는 각종구역을 관리하 는 소방, 경찰, 학교, 우편, 선거기관 및 각종 통계 를 산출하는 기관들이 국가기초구역을 단위로 하여 산출한 $\mathrm{DB}$ 를 의미한다. 국민들이 관심을 가지는 재 난, 치안, 교육 등의 정보를 근린생활단위로 제공받

\section{DB}

The DB by NBD of the building, land, buiness, residential data that people are intested and concerned to think spatially

\section{Sisting}

The DB by NBD of being produced by the spatial data agencies that

have existing spatial database

\section{Output}

The DB by NBD of being produced by the agencies of fire, police, school, mail, election activities which have the jurisdiction

* NDB : National Basic Districts

Figure 8. Data Types of Decision Support System based on National Basic Districts
게 되므로 지역생활에 대한 국민들의 관심도를 높 일 수 있다.

기본 $\mathrm{DB}$ 중 인구에 대한 정보는 통계청 인구센서 스 결과자료를 활용하는 방안과 주민등록(전산)정 보를 활용하는 방안이 있다. 그리고 건물에 대한 정 보는 건축행정정보시스템의 건축물대장 데이터나 국가공간정보통합체계의 건축물 통합 레이어에 포 함된 정보를 활용할 수 있다. 또한 토지정보의 경우 지적전산시스템의 토지대장 또는 토지적성자료를 활용하거나 한국토지정보시스템의 연속지적의 포함 정보를 활용할 수 있다. 입수 가능한 자료원을 통해 분야별로 설계한 기본DB데이터설계안은 Table 4 와 같다 [5]. 기본 $\mathrm{DB}$ 의 유지관리를 위해서 1 년에 1 번

Table 4. Database Design of the Decision Support System based on National Basic Districts

\begin{tabular}{|c|c|c|c|}
\hline Part & \multicolumn{2}{|c|}{ Field of National Basic Districts } & Source \\
\hline \multirow[b]{2}{*}{ Resident } & $\begin{array}{l}\text { Number of } \\
\text { Population }\end{array}$ & $\begin{array}{l}\text { - number of population } \\
\text { - population density }\end{array}$ & \multirow{2}{*}{$\begin{array}{l}\text { Resident } \\
\text { Registration } \\
\text { Informaion } \\
\text { System[21] }\end{array}$} \\
\hline & $\begin{array}{c}\text { State of } \\
\text { Residence }\end{array}$ & $\begin{array}{l}\text { - number of population } \\
\text { per state of } \\
\text { residence }\end{array}$ & \\
\hline Business & $\begin{array}{l}\text { Number } \\
\text { of Shop }\end{array}$ & $\begin{array}{l}\text { - number of shops } \\
\text { - shop density } \\
\text { - number of shop per } \\
\text { category of business }\end{array}$ & $\begin{array}{c}\text { Small } \\
\text { Enterprise } \\
\text { Development } \\
\text { Agency[24] }\end{array}$ \\
\hline \multirow{6}{*}{ Building } & \multirow{2}{*}{$\begin{array}{l}\text { Number } \\
\text { of } \\
\text { Building }\end{array}$} & - number of building & \multirow{6}{*}{$\begin{array}{c}\text { Korea } \\
\text { Address } \\
\text { Information } \\
\text { System[9] }\end{array}$} \\
\hline & & - main building annex & \\
\hline & $\begin{array}{l}\text { Building } \\
\text { Use }\end{array}$ & $\begin{array}{l}\text { - number of building } \\
\text { per building use }\end{array}$ & \\
\hline & $\begin{array}{l}\text { Date of } \\
\text { Completion } \\
\text { of Work }\end{array}$ & $\begin{array}{l}\text { - number of building } \\
\text { by year } \\
\text { - area of building by } \\
\text { year }\end{array}$ & \\
\hline & \multirow{2}{*}{$\begin{array}{l}\text { Number } \\
\text { of Floors }\end{array}$} & $\begin{array}{l}\text { - the number of } \\
\text { floors at ground } \\
\text { - average building } \\
\text { stories } \\
\text { - the maximun stoies } \\
\text { - the minimun stories } \\
\text { - the number of } \\
\text { building per floor }\end{array}$ & \\
\hline & & $\begin{array}{l}\text { - the number of floors } \\
\text { at underground } \\
\text { - average building } \\
\text { stories } \\
\text { - the maximun stories } \\
\text { - the minimun stories } \\
\text { - the number of } \\
\text { building per floor }\end{array}$ & \\
\hline Land & $\begin{array}{l}\text { Category } \\
\text { of Land }\end{array}$ & $\begin{array}{l}\text { - the number of lots } \\
\text { - total area } \\
\text { - the number of lots } \\
\text { per land objectivities } \\
\text { - the total area per } \\
\text { land objectivities }\end{array}$ & $\begin{array}{c}\text { Korea } \\
\text { Land } \\
\text { Information } \\
\text { System[19] }\end{array}$ \\
\hline
\end{tabular}


갱신하는 것을 원칙으로 하며 해당년도의 연앙(7월 1 일자)을 기준으로 한다. 수급한 정보를 기준년도 기초구역 데이터로 가공하여 저장하며 기초구역단 위 시계열 의사결정지원이 가능하도록 한다.

\section{3 국가기초구역기반 의사결정지원시스템 구축}

국가기초구역기반 의사결정지원시스템의 서비스 는 공공부문과 민간부문으로 구분하여 서비스 하며, 이는 행정정보의 공개 여부에 따라 구분한다. 의사 결정지원시스템의 서비스 개념은 기본적으로 기초 구역 기반의 공간DB에 속성 $\mathrm{DB}$ 를 결합하고, 이를 각종 행정자료 및 통계자료 등과 결합하여 제공하 는 것이다. 이에 가장 활용도가 높을 것으로 예상되 는 서비스에 대하여 사용자 편의성 및 활용도를 높 이기 위해 서비스 모델을 발굴·제공하도록 한다. 또 한 각종 스마트 기기에 대한 서비스를 수용함으로 써 사용자의 참여도를 극대화하는 것을 목표로 한 다. 국가기초구역기반 의사결정지원을 위한 목표시 스템 구성도는 Figure 9와 같다.

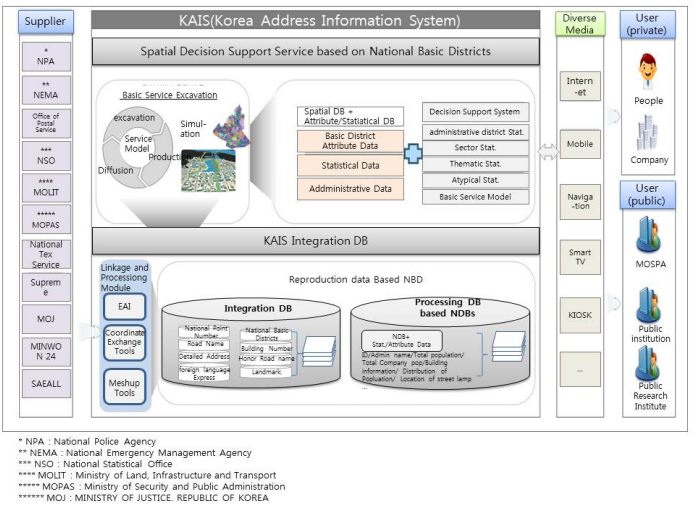

Figure 9. Concept of the Decision Support Service based on National Basic Districts

국가기초구역기반 의사결정지원시스템은 Figure 10 에 그려진 것 같이 지속적인 최신 $\mathrm{DB}$ 통합을 위 해 각 시스템 간 분산배치통합방식을 취하도록 한 다. 수집된 데이터는 검증, 가공과정을 거쳐 자료교 환체계를 통해 데이터를 배포하는 프로세스를 갖추 도록 한다.

기존 공간데이터를 국가기초구역단위 데이터로 가공하기 위한 연계모듈은 데이터설계, 연계모듈로 변환, 변환DB저장 및 관리의 과정을 거쳐 시스템에
저장되도록 설계하며 그 프로세스는 Figure 11과 같다.

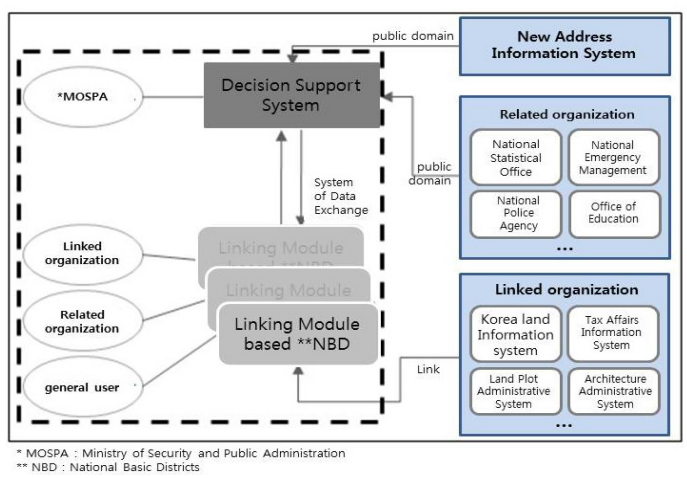

Figure 10. Concept of distributed layout for the Decision Support Service based on National Basic

Districts

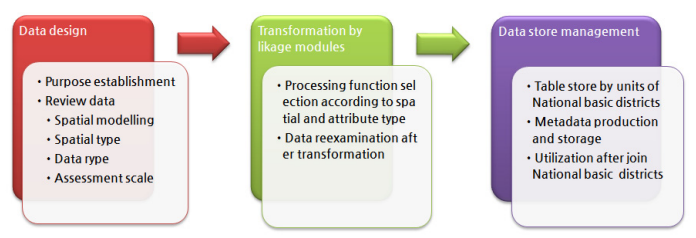

Figure 11. Data Processing Process using Linkage Modules of the Decision Support System based on National Basic Districts

국가기초구역기반 의사결정지원시스템의 어플리 케이션은 사용자가 기초구역단위로 구축된 다양한 데이터를 쉽게 의미있는 정보로 활용할 수 있도록 돕는 것이다. 공간분석서비스를 통해 일정 영역에 대한 교집합, 합집합, 차집합 등 영역 연산을 위한 공간질의 기능을 제공하고 사용자 정의 주제어 및 연관 주제어 등 키워드를 공개하며, 사용자가 원하 는 주제어로 키워드를 정의할 수 있으며, 연관 주제 어와의 접목으로 다양한 공간분석 기능을 제공한다. 또한 시계열정보를 활용하여 쉽게 변하지 않는 기 초구역을 활용한 시계열분석이 가능하도록 한다. 통 계지도서비스를 여러 주제, 사용자정의 주제별로 제 공하는 모듈을 개발하고 출력, 다운로드 등의 기능 을 강화한다. 사용자참여기능을 제공하여 사용자가 보유하고 있거나 생성한 데이터를 중첩하여 매쉬업 
하는 서비스를 제공하고 기본단위활용의 의미를 극 대화한다.

\section{4 안전행정부 의사결정지원을 위한 서비스 발굴}

안전행정부의 수요를 고려하여 기초구역 기반으 로 제공 가능한 업무지원 서비스를 발굴하고, 기초 구역단위 의사결정지원서비스를 통해 자치단체 행 정업무의 개선에 활용하도록 한다.

단계별 서비스 모델 도출을 위해 행정안전부의 2012년 업무추진계획을 토대로 키워드를 도출하고 업무영역에 대한 우선순위를 선정하였다. 분석결과 1순위 업무영역은 복지여성, 보건위생, 교통, 환경이 었으며 2순위 업무영역은 축산, 산림, 농업, 수산, 상하수도였다. 3순위 업무영역은 경제통상, 건축·주 택·건설, 세정·재정, 문화관광으로 나타났다.

업무영역에 대한 우선순위에 따라 행정안전부의 공간적 의사결정지원을 위한 서비스모듈을 구성하 도록 한다. 현행 업무에서의 정보 활용 현황 및 정 보요구의 분석을 통해 해당 분야의 의사결정지원서 비스의 요구를 분석하고 서비스모델을 설계한다. 서 비스모델은 서비스모델 시나리오 도출, 분석에 필요 한 공간정보 및 행정정보 목록화, 필요한 정보별 수 급 및 가공방안 설계, 시나리오 적용을 위한 분석 알고리즘 설계를 거쳐 분석모듈의 기능을 설계 한 다 [23].

\section{5. 결론 및 과제}

본 연구는 2014년부터 본격적으로 도입될 국가기 초구역의 활용전략으로서 국가기초구역기반 의사결 정지원시스템 구축전략 도출을 목표로 하였다.

국가GIS사업과 전자정부추진 등으로 공간적 의 사결정지원시스템의 구성요소인 데이터베이스, 지 식베이스, 업무모델이 갖추어져 있음에도 공간적 의 사결정지원시스템 구축에 어려움이 있는 이유는 체 계적인 자료 축적의 한계가 있음이 확인되었다.

국가기초구역의 특성과 국가기초구역관리시스템 의 아키텍쳐를 고려하여 국가기초구역기반 의사결 정지원시스템 구축전략의 방향을 정립하였다. 국가 기초구역기반 의사결정지원서비스구축 전략은 제 도, 데이터 및 시스템, 서비스의 3 분야로 나누어 제 안하였다.

제도적 측면에서 도로명주소법을 개정하여 국가
기초구역단위 데이터 산출규정 및 저장을 의무화할 필요가 있었다. 데이터 및 서비스 측면에서는 국가 기초구역단위의 인구정보, 건축물정보, 토지정보, 사업체정보 등을 나타내는 기본 $\mathrm{DB}$ 를 구축하고, 연 계 및 변환모듈을 이용한 기존 공간DB구축, 국가기 초구역 이용기관에서 산출되는 산출 $\mathrm{DB}$ 를 구축하도 록 제안하였다. 국가기초구역기반 의사결정지원시 스템의 서비스모델 측면에서는 안전행정부 업무분 석을 통해 복지, 안전 키워드를 도출하고 이를 모델 화하는 계획을 수립하였다.

국가기초구역단위 의사결정지원시스템이 최소공 간단위를 이용한 의사결정지원이라는 취지를 살려 나가기 위해서는 국가기초구역단위로 구축되는 속 성자료의 시계열적 관리가 가능한 시공간데이터베 이스(Spatial-Temporal Database)[33] 도입을 고려 할 필요가 있다. 이를 통해 국가기초구역단위 속성 정보의 변경이력을 통합적으로 관리하고 데이터베 이스의 무결성을 강화할 수 있을 것이다. 또한 국가 기초구역 의사결정지원시스템의 수요자 범위를 민 간, 안전행정부 내부, 정부부처, 지자체, 연구자 등 으로 다양화하여 시스템의 활용범위를 확장시키는 전략을 모색해야 할 것이다. 빅데이터의 활용가치가 높아진 스마트사회에서 국가기초구역기반 의사결정 지원시스템은 국가기초구역기반 데이터의 산출과 활용을 극대화하는 플랫폼이 될 수 있을 것으로 예 견된다.

\section{참고문헌}

[1] Architectural Administration Information system. http://www.eais.go.kr/

[2] Electronics and Telecommunications Research Institute , 2011, A Study of current situation of the Land Subdivision guideline for National Basic Districts

[3] Frada, B; Clyde W. H. 2008, Handbook on Decision Support Systems 1, Springer Link.

[4] Im. E. S. 2001, Spatial Decision Support Systems for Solid Waste Management : The Vehicle Routing-Scheduling for Waste Collection and the Site Selection of Incinerator, The graduate school of Konkuk University, Seoul, a doctoral dissertation. 
[5] Kang, Y. O. 2008, 2007 National GIS Support research - The research of basic statistics districts for the accumulation of Administrative and statistics data, Ministry of Land, Transport and Maritime Affairs.

[6] Kang, Y. O ; Jo, S. H. 2012, A Comparative Studyon Methods of Delimitating National Basic Districts, Journal of the Korean Cartographic Association, 12(1) : 113 127

[7] Kim, D. J. 2010, Urbansim Trends in Development and Topics, Planning and Policy, 348, 106-111.

[8] Kim, K. Y ; Choi, E. Y. 2011, The Roles of U.S. Census Bureau to the Development of GIS , Journal of the Korean Cartographic Association, 11(3):53-68.

[9] Choe, B. N; Im, E. S; Kim, K. 2007, The Strategies of Developing the Korea Planning Support System, The Journal of GIS Association of Korea, 15(4), 371-383.

[10] Korea Information Research and Development Institute, 2012, New Address Information System maintenance project Application Definition report.

[11] Korea Information Research and Development Institute, 2012, New Address Information System maintenance project Function Map.

[12] Lee, G. H; Shin, J. Y; Shin, S. H. 2010, The Research on the Location Suitability Analysis of the City Museum Using the GIS-Based Multicriteria Decision Analysis: A Case Study of Incheon, Journal of the Korean Urban Geographical Society, 13(3);89-105.

[13] Lee, H. Y; Lim, M. H; Yang, J. H. 2002, An Application to the Developing a Web-based Marketing Decision Making Support System = A Study on The Web-based System for Effectively Estimating Customer Utility, KOREAN MANAGEMENT REVIEW, 7(3) : 1787-1808.

[14] Lee, H. Y; Park, S. S. 2004, Application of a Decision Support System for Total Maximum Daily Loads, Journal of Korean Society on
Water Environment , 20(2);151-156.

[15] Lim, H. R. 2009, NGIS Final Report, The Korean Society For Geospatial Information System, September,159-163.

[16] Ministry of Land, Transport and Maritime Affairs, 2012, Korea Planning Support Systems (KOPSS) Final Report.

[17] Ministry of Land, Transport and Maritime Affairs, 2011, Korea Planning Support Systems (KOPSS) Concept and Spread Plan.

[18] Ministry of Land, Transport and Maritime Affairs, 2012, Korea Planning Support Systems (KOPSS) Final Report.

[19] Ministry of Land, Transport and Maritime Affairs, 2010, NGIS White Paper.

[20] Ministry of Land, Transport and Maritime Affairs, 2012, KLIS table definition map

[21] Ministry of Public Administration and Security, 2012, Tasks plan.

[22] Ministry of Public Administration and Security, 2012, The administrative items of resident registration information system data field

[23] Ministry of Public Administration and Security, 2008, Strategy Planning for linking and using both administrative and spatial information for local government services.

[24] National Information Society Agency, 2013, The bigdata era opening the new era.

[25] Openmate, 2012, Table definition of Market data, Openmake Ltd.

[26] Park, H. K; Keong, J. 1996, Decision Support Systems, Kyungmunsa.

[27] Park, J. M; Hwang, C. S. 2005, A Design and Practical Use of Spatial Data Warehouse for Spatial Decision Making, The Journal of GIS Association of Korea, 13(3), 239-252.

[28] Park, K. Y; Ahn, S. S ; Lee, H. Y. 2006, A studyon benefit segmentatin using decision support system (DSS) infood service industry, Journal of Tourism and Leisure Research, 18(1):103-120.

[29] Seoul Open Data Portal.http://data.seoul.go.kr/

[30] Shimizu, T; Marly Monteiro de C; Fernando, J. 
B. L. 2006, Strategic Alignment Process and Decision Support Systems: Theory and Case Studies, IRMPress.

[31] The Common Resources Portal. http://www.data.go.kr/

[32] The United Kingdom Common Data Portal . http://data.gov.uk/

[33] Timos, K. S; Manolis, K; Andrew, F; Stéphane, G; Ralf, H. G ; Christian , J ; Nikos, A. L; Yannis, M; Enrico, N; Barbara, P; Babis, T; Nectaria, T; Hans-Jörg, S; Michel, O. S.2003, Spatio-Temporal Databases - The CHOROCHRONOS Approach, Springer Link.
[34] United States Common Resources Portal. http://www.data.gov/

논문접수 : 2013.03.10

수 정 일 : 2013.04.23

심사완료 : 2013.04.25 